\title{
Rate equation and scaling for fragmentation with mass loss
}

\author{
Boyd F. Edwards, Mao Cai, and Hongtao Han \\ Department of Physics, West Virginia University, Morgantown, West Virginia 26506
}

(Received 22 February 1989)

\begin{abstract}
A linear rate equation describes fragmentation with continuous and discrete mass loss typified by consumption of porous reactive solids and two-phase heterogeneous solids. For a massdependent fragmentation rate $x^{\alpha}$ and a continuous-mass-loss rate $\epsilon x^{\gamma}, \sigma=\gamma-\alpha-1<0$ yields a "recession regime" where small particles lose mass continuously without breaking, $\sigma>0$ yields a "fragmentation regime" where all particles break, and $\sigma=0$ yields scaling for $\alpha>0$. Shattering for $\alpha<0$ and $\sigma \geq 0$ is runaway fragmentation producing an infinite number of particles in a finite time. Exact and asymptotic solutions, exponent relations, and connections with static percolation are found.
\end{abstract}

Fragmentation results from a variety of physical processes including erosion, polymer degradation, ${ }^{1}$ grinding, ${ }^{2}$ oxidation, ${ }^{3,4}$ and dissolution. In many of these processes, mass loss plays a crucial role. The goal of this Rapid Communication is to develop a theory of fragmentation with mass loss describing the time evolution of the particle mass distribution, and to identify universal and scaling behaviors in these systems.

The rate equation approach has proved fruitful for fragmentation in the absence of mass loss. A linear rate equation ${ }^{1,5}$ describes processes such as polymer degradation in which noninteracting particles break due to some corrosive chemical or electromagnetic agent present in the environment. A nonlinear rate equation ${ }^{5}$ describes fragmentation processes involving repeated particle collisions reminiscent of Smoluchowski aggregation. ${ }^{6,7}$

"Continuous" mass loss is essential to fragmentation during oxidation and dissolution of solid porous objects, and may play an important role in some polymer degradation processes. In these linear systems, an external reaction (such as oxidation) consumes solid mass at exposed particle surfaces, causing these surfaces to recede continuously. Reaction within the pores causes the pores to widen and fuse. Fragmentation occurs as final bridges between different parts of the same particle are consumed. Thus, surface recession is the cause of fragmentation in these linear systems. Experiments indicate that many such fragmentation events can occur during the oxidation of a single coal char particle. 4,8

We also consider "discrete" mass loss typified by reactions in two-phase heterogeneous solids containing isolated inclusions of an explosive phase imbedded within a much slower-reacting phase. Mass loss occurs continu- ously during surface recession of the slower-reacting phase. When surface recession exposes an explosive inclusion, discrete mass loss occurs as the mass in that inclusion is consumed instantaneously (compared to the consumption rate of the slow phase). At the same instant, fragmentation occurs in the slow-phase regions surrounding the explosive inclusion. In this way, discrete mass loss accounts for any mass loss that occurs during fragmentation events.

The goal of this paper is to describe fragmentation with continuous and discrete mass loss using a linear rate equation approach. To date, knowledge about such fragmentation has been drawn only from numerical simulations of specific particle morphologies. ${ }^{3,4}$ Our analysis identifies regimes relevant to a rich spectrum of particle morphologies and reaction conditions, including a scaling regime where the fragmentation and surface recession properties are independent of the particle mass. Furthermore, we obtain exact and asymptotic solutions including a solution corresponding with one-dimensional static percolation.

The appropriate linear rate equation

$$
\begin{aligned}
\frac{\partial n(x, t)}{\partial t}= & -a(x) n(x, t)+\int_{x}^{\infty} a(y) \bar{b}(x \mid y) n(y, t) d y \\
& +\frac{\partial}{\partial x}[c(x) n(x, t)]
\end{aligned}
$$

involves the particle mass distribution $n(x, t)$, a fragmentation rate $a(x)$, a distribution $\bar{b}(x \mid y)$ of daughterparticle masses $x$ spawned by the fragmentation of a parent particle of mass $y$, and a continuous-mass-loss rate $c(x)$, the essential new feature of the theory. Equation (1) follows from the number of particles entering the mass range $[x, x+d x]$ during an elapsed time $d t$,

$$
\begin{aligned}
d n(x, t) d x= & -a(x) d t n(x, t) d x+\int_{x}^{\infty} a(y) d t n(y, t) d y \bar{b}(x \mid y) d x \\
& +n(x+d x, t) d x c(x+d x) d t / d x-n(x, t) d x c(x) d t / d x .
\end{aligned}
$$

Here, $n(x, t) d x$ is the number of particles in the range at time $t, a(x) d t$ is the fraction of this number that break in an elapsed time $d t, c(x) d t / d x$ is the fraction of this number that lose sufficient mass to leave the range in an elapsed time $d t$, and $\bar{b}(x \mid y) d x$ is the probability that a daughter spawned by a particle of mass $y$ lies in the range.
Thus, the terms on the right-hand side account for (i) fragmentation of particles in the range, (ii) fragmentation producing particles in the range, (iii) continuous mass loss producing particles in the range, and (iv) continuous mass loss causing particles to leave the range.

Defining a discrete loss fraction $\lambda$ as the mass fraction 
lost from a parent particle at the instant of fragmentation requires a $\lambda$-specific daughter-particle distribution $b(\lambda$; $x \mid y)$ and a distribution $\Phi(\lambda, y)$ of discrete loss fractions $\lambda$ resulting from the fragmentation of a parent particle of mass $y$, with $\int_{0}^{1} \Phi(\lambda, y) d \lambda=1$. A relation ensuring that the total mass of daughter particles equals the reduced parent mass, $\int y x b(\lambda ; x \mid y) d x=(1-\lambda) y$, immediately yields a normalization condition

$$
\int_{0}^{y} x \bar{b}(x \mid y) d x=[1-\bar{\lambda}(y)] y,
$$

where $\quad \bar{b}(x \mid y)=\int_{0}^{1} b(\lambda ; x \mid y) \Phi(\lambda, y) d \lambda$ and $\bar{\lambda}(y)$ $=\int_{0}^{1} \lambda \Phi(\lambda, y) d \lambda$ are averaged quantities. The number of daughter particles, $\int_{b} b(\lambda ; x \mid y) d x=\mathcal{N}(\lambda, y)$, yields an average

$$
\int_{0}^{y} \bar{b}(x \mid y) d x=\overline{\mathcal{N}}(y) .
$$

Multiplying Eq. (1) by $x$ and integrating yields the rate of change of total mass,

$$
\dot{M}=-\int_{0}^{\infty}[x \bar{\lambda}(x) a(x)+c(x)] n(x, t) d x .
$$

The familiar equations for mass-conserving fragmentation $^{1,5}$ can be recovered by setting $c(x)=0$ and $\Phi(\lambda$, $y)=2 \delta(\lambda)$, which ensure zero continuous and zero discrete mass loss.

A spatially discrete random bond annihilation process yields a connection with static percolation. Initially, all bonds (unit line segments) on a line are deemed as occupied, forming a chain of infinite length. As occupied bonds are randomly annihilated to form smaller chains, the appropriate discrete form of Eq. (1) (Ref. 9)

$$
\frac{d n_{x}}{d t}=-x n_{x}+2 \sum_{y=x+1}^{\infty} n_{y}
$$

yields the number $n_{x}(t)$ of chains of length $x$ at time $t$ per bond, with $x$ restricted to positive integer values. Equation (5) is similar to a rate equation for infinite-dimensional percolation on the Bethe lattice. ${ }^{10}$ The random bond annihilation problem is equivalent to static onedimensional bond percolation with a bond occupation probability $p=e^{-t}$. Indeed, an exact solution $n_{x}(t)$ $=\left(1-e^{-t}\right)^{2} e^{-x t}$ to Eq. (5) agrees with the known solution ${ }^{11} n_{x}=(1-p)^{2} p^{x}$ for one-dimensional percolation. This correspondence provides a specific confirmation of Eq. (1) involving discrete and continuous mass loss, and shows the power of the rate equation approach for onedimensional percolation.

To identify physical regimes implied by the power-law rates $a(x)=x^{\alpha}$ and $c(x)=\epsilon x^{\gamma}$, we study the time evolution of a large initial number $N_{0}$ of particles each of initial mass $m_{0}$ subject to surface recession and fragmentation. Recalling the definitions of $c(x)$ and $a(x)$ allows us to use $c(m)=-\dot{m}$ and $\dot{N}=-a(m) N$ to find the timedependent mass $m(t)=m_{0}\left[1+(\gamma-1) \epsilon m_{0}^{\gamma^{-1}} t\right]^{(1-\gamma)^{-1}}$ of each unbroken particle and the corresponding number $N(t)=N_{0} \exp \left\{-\left[\left(m / m_{0}\right)^{-\sigma}-1\right] / \sigma \epsilon m_{0}^{\sigma}\right\}$ of such particles, where $\sigma=\gamma-\alpha-1$. Since $N N_{0}^{-1} a(m) d t \equiv g(t$, $\left.m_{0}\right) d t$ is the fraction of unbroken particles that break between time $t$ and time $t+d t, g\left(t, m_{0}\right)$ is the distribution of lifetimes of particles of initial mass $m_{0}$.

It is convenient to introduce a continuous loss fraction $\lambda_{c}=1-m(t) / m_{0}$, defined as the fraction of the mass of a particle lost to surface recession during its lifetime $t$. By setting $g\left(t, m_{0}\right) d t=\phi_{c}\left(\lambda_{c}, m_{0}\right) d \lambda_{c}$, we obtain the distribution of continuous loss fractions for particles of initial mass $y$ which break before losing all their mass,

$$
\phi_{c}\left(\lambda_{c}, y\right)=\frac{\left(1-\lambda_{c}\right)^{-(\sigma+1)}}{\epsilon y^{\sigma}} \exp \left(-\frac{\left(1-\lambda_{c}\right)^{-\sigma}-1}{\sigma \epsilon y^{\sigma}}\right) .
$$

Thus, the cumulative distribution $F\left(\lambda_{c}, y\right)=\int_{0}^{\lambda_{c}} \phi_{c}\left(\tilde{\lambda}_{c}\right.$, $y) d \tilde{\lambda}_{c}$ gives the probability that a particle of initial mass $y$ will have a continuous loss fraction between zero and $\lambda_{c}$.

The total fragmentation probability $F(1, y)$, obtained by direct integration, finally allows us to identify physical regimes. In a fragmentation regime with $\sigma>0$ and $F(1, y)=1$, particles are guaranteed to break before losing all their mass to surface recession. In a recession regime with $\sigma<0$ and $F(1, y)=1-\exp (-y|\sigma| /|\sigma| \epsilon)$, particles with initial masses much smaller than a cutoff mass $y_{c}=(|\sigma| \epsilon)^{1 /|\sigma|}$ lose all their mass without fragmentation. Thus, the recession regime is relevant experimentally when pore sizes have a minimum or when the consumptive reaction only partially penetrates the pores. ${ }^{3,4}$ In summary, the power-law rates yield a long-time regime governed by fragmentation for $\sigma>0$ and by surface recession for $\sigma<0$. The above procedure could easily yield analogous results for nonpower-law rates, if needed for comparison with experiments.

By introducing a $\delta$ function to allow for the possibility of losing all mass without fragmentation, we obtain a total continuous loss fraction distribution;

$$
\Phi_{c}\left(\lambda_{c}, y\right)=\phi_{c}\left(\lambda_{c}, y\right)+2[1-F(1, y)] \delta\left(\lambda_{c}-1\right),
$$

where $\int_{0}^{1} \Phi_{c}\left(\lambda_{c}, y\right) d \lambda_{c}=1$.

A small- $\lambda_{c}$ approximation $\phi_{c}\left(\lambda_{c}, y\right)=\left(\epsilon y^{\sigma}\right)^{-1}$ $x \exp \left(-\lambda_{c} / \epsilon y^{\sigma}\right)$ yields the average value $\bar{\lambda}_{c}(y)=\int_{0}^{1} \lambda_{c}$ $\times \Phi_{c}\left(\lambda_{c}, y\right) d \lambda_{c}=\epsilon y^{\sigma}$. Thus, in the fragmentation regime, small particles lose small fractions of their mass between fragmentation events, indicating an abundance of small pores. In the recession regime, the large particles lose small mass fractions between fragmentation events, indicating an abundance of large pores. For $\sigma=0$, the massindependent result $\bar{\lambda}_{c}(y)=\epsilon(1+\epsilon)^{-1}$ [from Eq. (6)] indicates a uniform pore-size distribution. Thus, the powerlaw rates describe a broad spectrum of physical systems, with values of $\sigma$ reflecting the pore-size distribution and the extent of pore penetration.

For $\bar{b}(x \mid y)=2 / y, \alpha=1, \gamma=0$ (and $\sigma=-2$ ), an explicit solution $n(x, t)=t^{2} \exp \left(-x t-\epsilon t^{2} / 2\right)$ to Eq. (1) illustrates that continuous-mass loss (proportional to $\epsilon$ ) is relevant at large $t$ when the particles are small, as expected for the recession regime.

Scale-invariant "scaling" solutions of partial differential equations hold great interest because of evidence ${ }^{6,12}$ that large classes of general solutions tend to scaling solutions after initial transients decay away. Invariance of Eq. (1) under the scale transformation $x=s x^{*}, t=s^{\phi} t^{*}$, and $n(x, t)=n\left(s x^{*}, s^{\phi} t^{*}\right)=s^{*} n^{*}\left(x^{*}, t^{*}\right)$ requires $\bar{b}(x \mid y)$ 
$=h(x / y) / y($ Ref. 5), $\sigma=\gamma-\alpha-1=0, \phi=-\alpha$, and a form for scaling solutions,

$$
n(x, t)=t^{\delta} f\left(x t^{z}\right),
$$

with scaling exponents $\delta=-\psi / \alpha$ and $z=1 / \alpha$. Scaleinvariant forms of Eqs. (1)-(3) involve mass-independent $\bar{\lambda}, \overline{\mathcal{N}}$, and daughter-fraction distribution $h(r)$;

$$
\begin{aligned}
& \frac{\partial n(x, t)}{\partial t}=-x^{\alpha} n(x, t)+\int_{x}^{\infty} y^{\alpha-1} h(x / y) n(y, t) d y \\
&+\epsilon \frac{\partial}{\partial x}\left[x^{\alpha+1} n(x, t)\right], \\
& \int_{0}^{1} r h(r) d r=1-\bar{\lambda}, \\
& \int_{0}^{1} h(r) d r=\overline{\mathcal{N}} .
\end{aligned}
$$

Hence, all properties of fragmentation and surface recession become independent of the particle mass for $\sigma=0$, thus reflecting the essence of scaling.

The scaling form [Eq. (8)] implies that the typical particle mass scales as $t^{-z}$. Thus, for $\alpha=1 / z>0$, this typical mass decreases with time as expected. Since this typical mass increases with time for $\alpha<0$, scaling is absent for $\alpha<0$. For mass-conserving fragmentation with $\alpha<0$, runaway fragmentation rates for the smallest particles result in an effectively infinite number of fragmentation events in a finite time, or "shattering," 1,5 thereby yielding unexpected mass loss to a phase of zero-mass particles. For fragmentation with mass loss and $\alpha<0$, the recession regime precludes runaway fragmentation since the smallest particles lose all their mass without breaking. The fragmentation and scaling regimes and discrete mass loss allow runaway fragmentation for $\alpha<0$, but might preclude the unexpected mass loss normally associated with shattering. ${ }^{9}$

For $\alpha>0$ and $\sigma=0$, we argue ${ }^{9}$ that discrete mass loss allows scaling, whereas continuous-mass loss violates scaling at large values of the scaling variable $\xi=x t^{2}$. For the power-law decay $h(r) \sim r^{v}$ as $r \rightarrow 0$, the small- $\xi$ asymptotic form ${ }^{9} f(\xi) \sim \xi^{v}$ is valid for both types of mass loss, whereas the large- $\xi$ form $f(\xi) \sim \xi^{h(1)-\delta a} \exp \left(-\xi^{\alpha}\right)$ is valid only for discrete mass loss $(\epsilon=0)$.

That the total mass $M=\int_{0}^{\infty} x n(x, t) d x=t^{\delta-2 z} \rho_{1}$ must decrease with time [with $\rho_{\mu}=\int_{0}^{\infty} \xi^{\mu} f(\xi) d \xi$ ] implies the scaling-exponent inequality ${ }^{3} \delta<2 z$. More usefully, Eq. (4) implies a direct relation with the observable mass loss parameters $\bar{\lambda}$ and $\epsilon$;

$$
2 z-\delta=(\bar{\lambda}+\epsilon) \rho_{1}^{-1} \rho_{1+1 / z} .
$$

This relation is approximate for finite $\epsilon$ (because of the violation of scaling at large $\xi$ ) and is exact for finite $\bar{\lambda}$ as $\epsilon \rightarrow 0$. In Ref. 5, since Eq. (11) satisfies Eq. (3) only if $\omega=1 / \lambda$, Eq. (13) [with $a=(\omega \lambda)^{-1 / \lambda}=1$ ] agrees with our large- $\xi$ asymptotic form when mass is conserved, that is, when $\delta=2 z$.

Exact solutions, ${ }^{9}$ easily verified by direct substitution, can be obtained for an intrinsically discrete loss process $(\epsilon=0)$ with a distribution $h(r)=(v+2)(1-\bar{\lambda}) r^{v}$ satisfying Eq. (10) for $v>-2$. The scaling solution $f(\xi)$ $=\xi^{v} \exp \left(-\xi^{\alpha}\right)$ with $\delta \alpha=(v+2)(1-\bar{\lambda})-v \leq 2$ agrees with the asymptotic scaling forms and yields $n(x, t)$ $=x^{v} t^{(v+2)(1-\bar{\lambda}) / a} \exp \left(-x^{a} t\right)$. This scaling solution is valid for all nonzero $t$, in contrast with scaling solutions for coagulation problems, ${ }^{6,12}$ which are often valid only asymptotically for large $t$. The corresponding general solution of Eq. (9) for arbitrary initial conditions,

$$
\begin{aligned}
n(x, t)=x^{v} \exp \left(-x^{\alpha} t\right) \int_{0}^{\infty} & A(s)(1+t / s)^{(v+2)(1-\bar{\lambda}) / \alpha} \\
& \times \exp \left(-s x^{\alpha}\right) d s,
\end{aligned}
$$

agrees with the scaling solution for large $t$ and fixed $x$. The average number of fragments from Eq. (11), $\overline{\mathcal{N}}=(v+2)(1-\bar{\lambda}) /(v+1)$, yields binary fragmentation only for $v=0$ and $\bar{\lambda}=0$. Fragmentation events yielding large daughter fractions $r>1-\bar{\lambda}$ require $\lambda<\bar{\lambda}$, whereas $\lambda>\bar{\lambda}$ is typical otherwise.

We have presented essential elements of a comprehensive theory of linear fragmentation accompanied by discrete and continuous-mass loss. The nature of solutions in nonscaling and shattering regimes deserves further study. Experimental tests of the theory would be helpful. It would be interesting to extend the theory to growth phenomena such as deposition, where mass gain by particles (rather than collisions between particles) leads to coalescence, and where $c(x)<0$. Whether a useful rate equation exists for static percolation in finite dimensions greater than unity is an interesting open question.

We gratefully acknowledge stimulating discussions with Alan Kerstein and Richard Treat, and support from the West Virginia University Energy and Water Research Center, the Pittsburgh Supercomputing Center, and Department of Energy Grant No. DE-FG22-89PC89791.
${ }^{1}$ E. D. McGrady and R. M. Ziff, Phys. Rev. Lett. 58, 892 (1987); R. M. Ziff and E. D. McGrady, Macromolecules 19, 2513 (1986); J. Phys. A 18, 3027 (1985).

${ }^{2}$ A. R. Kerstein (unpublished).

${ }^{3}$ M. Sahimi and T. T. Tsotsis, Phys. Rev. Lett. 59, 888 (1987).

${ }^{4}$ D. Dunn-Rankin and A. R. Kerstein, Combust. Flame 69, 193 (1987), and references therein; A. R. Kerstein and B. F. Edwards, Chem. Eng. Sci. 42, 1629 (1987).

${ }^{5} Z$. Cheng and S. Redner, Phys. Rev. Lett. 60, 2450 (1988).

${ }^{6}$ A. Meesters and M. H. Ernst, J. Colloid Interface Sci. 119, 576 (1987); G. W. Mulholland and H. R. Baum, Phys. Rev. Lett. 45, 761 (1980).

${ }^{7}$ T. Vicsek and F. Family, Phys. Rev. Lett. 52, 1669 (1984); T.
Vicsek, P. Meakin, and F. Family, Phys. Rev. A 32, 1122 (1985); F. Family, P. Meakin, and T. Vicsek, J. Chem. Phys. 83, 4144 (1985).

${ }^{8}$ C. A. Sundback, J. M. Beér, and A. F. Sarofim, in Proceedings of the Twentieth International Symposium on Combustion (The Combustion Institute, Pittsburgh, 1985), p. 1495.

${ }^{9}$ M. Cai, B. F. Edwards, and H. Han (unpublished).

${ }^{10}$ A. R. Kerstein, J. Phys. A 22, 3371 (1989).

"D. Stauffer, Introduction to Percolation Theory (Taylor and Francis, Philadelphia, 1985), Chap. 2.

${ }^{12}$ F. Family, P. Meakin, and J. M. Deutch, Phys. Rev. Lett. 57, 727 (1986). 